\title{
Report of a workshop sponsored by the European Organisation for Research and Treatment of Cancer (EORTC) on the treatment of primary malignant brain tumours $\star$
}

\author{
J Hildebrand, D G T Thomas
}

\section{High grade glioma}

The vast majority of high grade gliomas are glioblastomas or anaplastic astrocytomas. The former are twice as common as the latter type of primary brain tumour. Surgery is an important step in the management of these patients both to obtain material for a definite histological diagnosis and to achieve tumour resection. Radiation therapy consisting of 60 Gy delivered in six weeks prolongs the median survival by between $50-100 \%$, but does not appear to significantly increase the two year survival rate.

Attempts to potentiate radiation therapy through the use of various radio-sensitisers of anoxic cells have failed so far, and no advantage has yet been demonstrated of high dose fractionation techniques. The value of interstitial radiation therapy using $I^{125}$ is currently being tested in a randomised trial.

Nitrosoureas are the most effective single chemotherapy agents for tumour recurrence, achieving up to a $50 \%$ response rate in terms of clinical improvement or stabilisation lasting six to nine months. However, total regression of contrast enhanced tumour area on computer tomography (CT) scan is seen in less than $20 \%$ of cases. Procarbazine, aziridinylbenzoquinone (AZQ), cisplatinum and in childhood high grade gliomas, cyclophosphamide and vincristine are also active chemotherapy agents in brain tumours. No combination chemotherapy, however, has proved to be superior to single nitrosoureas therapy in prospective and randomised studies. Autologous bone marrow grafts allow a three to five fold increase of nitrosoureas doses in the treatment of brain tumours. At least six studies have been performed showing the feasibility of this technique but none of these has shown a superior clinical result compared with conventional chemotherapy techniques. Intracarotid delivery of chemotherapy agents is only useful for agents which rapidly cross the blood-brain barrier and has been used mainly for nitrosoureas and cisplatinum. Where the drug doses used for intra-carotid administration are equal to those used systemically the drug concentrations in the tumour concentration are significantly higher and the rate of remissions is probably increased with the further benefit that systemic toxicity is reduced. Unfortunately, central nervous system and eye toxicity limit the benefit of this method. It is possible that this technique of intra-carotid delivery will improve with the use of platinum derivatives.

The use of adjuvant chemotherapy, starting soon after operation may make a small improvement in results. BCNU increases survival rate at 18 and 24 months by about $15 \%$. CCNU, $\mathrm{VM}_{26}$ and 5-fluorouracil, CCNU plus dibromodulcitol and the combination of CCNU, procarbazine and vincristine (in astrocytomas) possibly increase the median survival. These questions are presently addressed by a number of different groups.

New studies include the use of $\beta$ interferon which appears to be efficacious in the short term, and other cytokines such as interleukin two alone or in combination with lymphocyte activated killer (LAK) cells. Combinations of calcium channel blockers, such as nimodipine, or hyaluronidase with conventional chemotherapeutic agents have shown promising initial results.

\section{Low grade astrocytoma}

Because low grade astrocytomas are relatively rare and lack universally agreed criteria for their histological definition, studies concerning their management are infrequent and also are difficult to assess. It is generally accepted that low grade astrocytomas correspond to grade two astrocytomas by the recent World Health Organisation classification, regardless of their appearance on neuroradiological examination performed with enhanced CT scan. Oligodendroglioma, a form of low grade glioma, which has recently been reported as being quite sensitive to chemotherapy using melphalan, must be considered as a separate entity.

The five year survival following surgery for patients with low grade astrocytoma ranges from $43-82 \%$. Whether radiation therapy should be given early or delayed until recurrence and also the most appropriate dose of radiotherapy both remain crucial and as yet unanswered questions. Several current trials are in progress addressing these issues.

Because low grade astrocytomas are better circumscribed tumours anatomically than high grade gliomas, on theoretical grounds interstitial radiotherapy should be a valuable treatment. As yet, prospective studies comparing interstitial radiation therapy combined with extracranial radiation therapy compared with extracranial radiation therapy are not available. 
Low grade astrocytomas are sensitive to drugs which are active in high grade glioma and the response rate in these lower grade tumours is usually higher. In addition, since low grade astrocytomas are less infiltrative they are more likely to respond to drugs such as glycophosphamide, which does not readily cross the blood-brain barrier.

\section{Primary brain lymphomas}

In patients without immunosuppression, due for exanple to transplantation or HIV infections, less than $2 \%$ of primary brain tumours are primary brain lymphomas. Only $1 \%$ of all non-Hodgkin's lymphomas originate in the brain. Although primary brain lymphomas are pathologically similar to systemic nonHodgkin's lymphomas, the response to therapy, particularly to radiation therapy, is less satisfactory. Unfortunately, despite the fact that primary brain lymphomas are confined to the central nervous system, a cure cannot be achieved with radiation therapy and/or chemotherapy and operation has only a diagnostic value.

The common radiation therapy dose of approximately $45 \mathrm{~Gy}$ is derived from the treatment of systemic non-Hodgkin's lymphomas. The optimal dose for primary brain lymphomas, however, remains to be determined. Radiation therapy has moved the median survival from a few months to over one year and the addition of chemotherapy has increased the median survival to about two years.

Various chemotherapy agents, singly or in combination, have been found effective. The most frequently used are daunorubicin, adriamycin, cylophosphamide, vincristine, high-dose methotrexate and corticosteroids. Several authors are currently testing the optimal doses and sequence of both radiation therapy and chemotherapy in primary brain lymphomas.

In HIV-related brain lymphoma the situation is quite different. Within the next few years in certain geographical areas this tumour may become the most common form of primary brain neoplasm. At present, in $90 \%$ of patients with this tumour treated with various radiotherapy programmes death is not due to the cerebral neoplasm but to other aspects of the disease.

\section{Medulloblastoma}

Medulloblastoma is the most common brain tumour in childhood. These tumours arise in the posterior fossa and are characterised by a rapid growth and a high proliferative cell fraction. The latter property makes them very sensitive to radiotherapy and chemotherapy.

Surgical removal followed by irradiation of the entire central nervous system yields an overall five year survival rate of over $50 \%$. Several high risk factors have been identified: the tumour extent and size $(>1.5 \mathrm{~cm}$ in diameter), age (under two years), and undifferentiated pathology. The most commonly used doses of radiotherapy are 50-55 Gy over the posterior fossa, 35-45 Gy to the rest of the brain and 25-30 Gy to the spine. The recent tendency, however, is to reduce these doses by $10 \mathrm{~Gy}$ in low risk patients, mainly to decrease central nervous system toxicity.

At recurrence, at least two-thirds of the patients will respond to chemotherapy which in the past has consisted mainly of a combination of nitrosoureas (often), vincristine and PBZ. More recently, cyclophosphamide and cisplatinum have been tested. Patients who respond to chemotherapy at recurrence have a longer and improved quality of survival.

The usefulness of adjuvant chemotherapy is not entirely resolved. The results of two large cooperative studies (the International Society for Paediatric Oncology, and the Children's Cancer Study Group) have indicated that benefit was restricted to high risk patients only. However, the chemotherapy used (CCNU and vincristine) may not have been the most effective. The role of adjuvant chemotherapy requires further assessment, as does the use of pre-irradiation chemotherapy. 